\title{
Pulmonologists and lung cancer: pivotal role in multidisciplinary approach
}

\author{
Mina Gaga', Jean-Paul Sculier ${ }^{2}$ and Klaus F. Rabe ${ }^{3}$
}

Affiliations: ${ }^{17}$ th Respiratory Medicine Dept, Athens Chest Hospital, Athens, Greece. ${ }^{2}$ Institut Jules Bordet, Brussels, Belgium. '3 Zentrum für Pneumologie und Thoraxchirurgie, Krankenhaus Großhansdorf, Großhansdorf, Germany.

Correspondence: M. Gaga, Athens Chest Hospital, 7th Respiratory Medicine Dept, 152 Mesogion Ave, Athens 11527, Greece. E-mail: minagagadyahoo.com

0 @ERSpublications

The pulmonologist plays a vital role in the multidisciplinary approach to lung cancer diagnosis and management http://ow.ly/oALXp

Lung cancer carries a huge disease burden: the death toll is high, the disease severely impacts the quality of life of patients and their families, and it is associated with considerable costs for patients and health systems. It remains the leading cause of cancer mortality in the world, with approximately 1.4 million deaths per year. Lung cancer morbidity and mortality needs to be reduced, mainly through implementation of prevention strategies (including smoking cessation, reduction of occupational exposure and lifestyle measures), and most importantly by early diagnosis and prompt and targeted treatment. The role of the pulmonologist is pivotal in the diagnosis, staging and management of lung cancer and this role is comprehensively described in the joint American Thoracic Society (ATS)/European Respiratory Society (ERS) statement published in the August issue of the American Journal of Respiratory and Critical Care Medicine [1].

Pulmonologists are responsible for, and involved with, lung cancer patients from their initial diagnosis and staging through treatment and re-staging. They are also involved in the management of comorbidities and, importantly, complications, either from the tumour itself or the treatment, as well palliative and end-of-life care. Due to their knowledge of lung physiology, they are the appropriate physicians to estimate respiratory reserve, which is crucial for management decisions, and are thus best qualified to deal with lung-related morbidity and respiratory failure. This is even more important since many patients with lung cancer have respiratory comorbidities that affect their performance status and need to be taken into account in management decisions, the most common comorbidity being chronic obstructive pulmonary disease.

Early and specific diagnosis is very important in lung cancer, and recent studies demonstrated important advances that promise to decrease death rates over time $[2,3]$. The National Lung Screening Trial (NLST), which included more than 50000 patients at high risk of lung cancer, showed a relative reduction of $20 \%$ in lung cancer mortality with low-dose computed tomography screening [3]. The evaluation of lung nodules and other abnormalities detected at screening or diagnostic work-up requires tissue sampling and accurate histological and molecular characterisation of the tumour $[4,5]$.

This characterisation is crucial as various lung cancer cell types respond very differently to treatment. Current standards of care for lung cancer assign treatment not only on standard histology but also on the presence of specific molecular markers, including the presence of epidermal growth factor receptor mutation and anaplastic lymphoma kinase translocation status for adenocarcinoma [6, 7]. Tissue sampling can be performed by the pulmonologist with the use of guided bronchoscopy procedures, such as electromagnetic navigation, endobronchial ultrasound and virtual bronchoscopic navigation [8-11]. Recent

Received: Aug 192013 | Accepted: Aug 282013

Conflict of interest: None declared.

Copyright (C)ER 2013 
studies have shown that endobronchial ultrasound-guided transbronchial needle aspiration and transoesophageal ultrasound are less invasive and at least as accurate as mediastinoscopy [12, 13]. Thus, pulmonary physicians have important roles in educating and advising patients about the benefits and risks of screening procedures, and in managing findings in those patients who participate in screening programmes, as well as in patients who present with radiological findings and symptoms suggestive of lung cancer [14].

Pulmonologists also fulfil an important role in lung cancer treatment. Lung cancer care varies widely from country to country and sometimes even within countries. In many European countries, the management of lung cancer, including chemotherapy, is provided by pulmonologists, whereas in others, similar to the USA, chemotherapy is prescribed by medical oncologists. Palliative endoscopic techniques such as stents and endobrochial laser therapy are also performed by pulmonologists. In all cases, physicians involved in lung cancer care should be specifically trained in thoracic oncology as this cancer behaves very differently from other cancers, such as breast, ovarian, colon or prostate carcinoma, and recent evidence suggests that multidisciplinary teams and high-volume centres provide better care and have better overall outcomes [15]. In order to standardise lung cancer care, the ERS has asked for the collaboration of the European Society for Thoracic Surgeons, the European Society for Medical Oncology and the European Society for Radiotherapy Oncology, and is currently already working on a syllabus and a curriculum in thoracic oncology [16, 17]. Following this, an examination leading to the European Diploma in Thoracic Oncology will be soon established. We firmly believe that this initiative will promote optimal patient care.

Optimal lung cancer care requires a multidisciplinary team of specialists who care for a significant number of patients on a regular basis $[15,18]$. Although recommended by scientific societies, multidisciplinary management is not yet uniformly available. Ideally, centres managing patients with thoracic malignancies should have a multidisciplinary thoracic oncology board to make decisions on individualised management, based on evidence-based data. The multidisciplinary team should ideally include representatives of all specialties involved in the management of thoracic malignancies: pulmonologists, thoracic surgeons, medical oncologists, radiation oncologists, pathologists, radiologists and nuclear medicine specialists, as well as nurses, pharmacists, physiologists and palliation specialists. Treatment plans using surgical, radiotherapeutic and pharmacological approaches should be reviewed by the entire team. Moreover, there is evidence that high-volume centres and multidisciplinary teams are more efficient in managing lung cancer patients than low-volume or non-multidisciplinary centres, by providing more complete staging and better adherence to guidelines, leading to increased survival $[15,18]$. Data supporting multidisciplinary teams are included in national guidelines for lung cancer centres under the coordination of chest physicians [19]. In many European countries and the USA, tumour boards are required for cancer programme accreditation but this is not the case everywhere.

Lung cancer care, however, is not only a matter of medical education and experience, or even a thoracic oncology board: it is also a matter of resources, medical tests for early diagnosis, prompt access to surgical and radiotherapy units, effective medications and psychological support. The recent ERS task force for "Quality management in lung cancer care" that assessed usual practice and the resources available in the countries of the European Union, as well as in those represented at the national level in the ERS, reports very important differences in the standards of care. Similar disparities in health and welfare costs have already been reported [20].

International societies such as the ATS and the ERS must liaise with health policy makers and push for acceptable and, ideally, uniform standards of care across all countries. The ERS has been working towards this end through various actions and yearly presidential summits that bring together healthcare providers, patients, policy makers and the pharmaceutical industry in order to discuss the unmet needs in current practice and possible ways to move forward $[21,22]$. With regards to lung cancer in particular, research into pathogenetic mechanisms and into new drugs is very important, and pulmonologists should be involved in these research projects. Moreover, all the aforementioned involved parties must ensure that research leading to effective and cost-effective medications will be supported, acknowledging that better medications are needed but also that healthcare systems must be sustainable in the long term, and healthcare costs can continue to be met [23]. The role of the pulmonologist in this complex patient management and decisionmaking process is pivotal. The need for provision of personalised care in each individual lung cancer patient requires the specific skills of pulmonary physicians described above. The requisite pathway leads through pulmonology fellowship programmes that should offer extensive training and experience in lung cancer management so that pulmonologists will be well qualified to manage all aspects of care of these patients. Highly trained and qualified pulmonologists can play a central role in multidisciplinary lung cancer management teams, and can contribute effectively to the improvement of lung cancer outcomes and quality of the lives of patients and their families, and the reduction of the cost and impact on society. 


\section{References}

1 Gaga M, Powell CA, Schraufnagel DE, et al. An American Thoracic Society and European Respiratory Society Statement: the role of the pulmonologist in the diagnosis and management of lung cancer. Am J Respir Crit Care Med 2013; 188: 503-507.

2 Kris MG, Benowitz SI, Adams S, et al. Clinical cancer advances 2010: annual report on progress against cancer from the American Society of Clinical Oncology. J Clin Oncol 2010; 28: 5327-5347.

3 The National Lung Screening Trial Research Team. Reduced lung-cancer mortality with low-dose computed tomographic screening. N Engl J Med 2011; 365: 395-409.

4 Schwarz C, Schönfeld N, Bittner RC, et al. Value of flexible bronchoscopy in the pre-operative work-up of solitary pulmonary nodules. Eur Respir J 2013; 41: 177-182.

5 Schuhmann M, Bostanci K, Bugalho A, et al. EBUS-guided cryo biopsies in peripheral pulmonary lesions: a feasibility study. Eur Respir J 2013 [In press DOI: 10.1183/09031936.00011313]

6 Scoccianti C, Vesin A, Martel G, et al. European Early Lung Cancer Consortium. Prognostic value of TP53, KRAS and EGFR mutations in nonsmall cell lung cancer: the EUELC cohort. Eur Respir J 2012; 40: 177-184.

7 Travis WD, Brambilla E, Noguchi M, et al. International Association for the Study of Lung Cancer/American Thoracic Society/European Respiratory Society International Multidisciplinary Classification of Lung Adenocarcinoma. J Thoracic Oncol 2011; 6: 244-285.

8 Hetzel J, Eberhardt R, Herth FJ, et al. Cryobiopsy increases the diagnostic yield of endobronchial biopsy: a multicentre trial. Eur Respir J 2012; 39: 685-690.

9 Steinfort DP, Russell PA, Tsui A, et al. Interobserver agreement in determining non-small cell lung cancer subtype in specimens acquired by EBUS-TBNA. Eur Respir J 2012; 40: 699-705.

10 Peschke A, Wiedemann B, Höffken G, et al. Forceps biopsy and suction catheter for sampling in pulmonary nodules and infiltrates. Eur Respir J 2012; 39: 1432-1436.

11 Herth FJ, Schuler H, Gompelmann D, et al. Endobronchial ultrasound-guided lymph node biopsy with transbronchial needle forceps: a pilot study. Eur Respir J 2012; 39: 373-377.

12 Adams K, Shah PL, Edmonds L, et al. Test performance of endobronchial ultrasound and transbronchial needle aspiration biopsy for mediastinal staging in patients with lung cancer: systematic review and meta-analysis. Thorax 2009; 64: 757-762.

13 Annema JT, van Meerbeeck JP, Rintoul RC, et al. Mediastinoscopy vs endosonography for mediastinal nodal staging of lung cancer: a randomized trial. JAMA 2010; 304: 2245-2252.

14 Horeweg N, van der Aalst CM, Vliegenthart R, et al. Volumetric computer tomography screening for lung cancer: three rounds of the NELSON trial. Eur Respir J 2013 [In press DOI: 10.1183/09031936.00197712].

15 Freeman RK, Van Woerkom JM, Vyverberg A, et al. The effect of a multidisciplinary thoracic malignancy conference on the treatment of patients with lung cancer. Eur J Cardiothorac Surg 2010; 38: 1-5.

16 Meert AP, Noel JL, Boffetta P, et al. Thoracic Oncology HERMES Task Force: Harmonised Education and Training of Thoracic Oncology Specialists in Europe. Breathe 2013; 9: 381-392.

17 Gamarra F, Boffetta P, De Ruysscher D, et al. Thoracic Oncology HERMES syllabus: setting the basis for thoracic oncology training in Europe. Eur Respir J 2013; 42: 568-571.

18 Blum T, Schonfeld N, Kollmeier J, et al. Lungenkrebs in Deutschland - zur Versorgungslage der Nation [Lung cancer in Germany - the current state of management]. Pneumologie 2011; 65: 7-18.

19 Goeckenjan G, Sitter H, Thomas M, et al. Prevention, diagnosis, therapy, and follow-up of lung cancer: interdisciplinary guideline of the German Respiratory Society and the German Cancer Society. Pneumologie 2011; 65: 39-59.

20 Sculier JP, Vansteenkiste J, Schonfeld N, et al. Thoracic oncology in Europe: the ERS action plan by the Thoracic Oncology Assembly. Eur Respir J 2010; 36: 1227-1228.

21 Rabe KF, Ward B. Uncovering and tackling Europe's hidden respiratory illness. Lancet 2012; 380: 623-624.

22 Sibille Y, Decramer M, Nicod LP, et al. Directing the future of lung health: the European Respiratory Roadmap. Eur Respir J 2011; 38: 502-506.

23 Borget I, Cadranel J, Pignon JP, et al. ERMETIC Collaborative Group. Cost-effectiveness of three strategies for secondline erlotinib initiation in nonsmall-cell lung cancer: the ERMETIC study part 3. Eur Respir J 2012; 39: 172-179. 